\title{
Peristomal skin complications: causes, effects, and treatments
}

\section{Kimberly Doctor \\ Dorin T Colibaseanu}

Section of Colon and Rectal Surgery, Mayo Clinic, Jacksonville, FL, USA
Correspondence: Dorin T Colibaseanu Section of Colon and Rectal Surgery, Mayo Clinic, 4500 San Pablo Road, Jacksonville, FL 32224, USA Email colibaseanu.dorin@mayo.edu
This article was published in the following Dove Press journal:

Chronic Wound Care Management and Research

19 December 2016

Number of times this article has been viewed

Abstract: Enterostomal formation remains a necessary part of multiple types of surgeries. Stomal difficulties can be a source of frustration for patients; however, a properly functioning stoma in a patient educated in its care can result in a highly functional individual, with a high quality of life, comparable to a person without a stoma. Correct surgical technique is vital to creating a stoma that is sufficiently everted, and in a good anatomical location. Loop ileostomies have a higher chance of complications, thus care in their formation is especially important. Systemic disease (inflammatory conditions, and autoimmune diseases especially) as well as local conditions (pyoderma gangrenosum, infections, and fistulas, among others) can be the causes for difficult-to-treat peristomal complications. Accurate diagnosis is essential in order to be able to address the underlying disease. Choosing the appropriate products to care for the stoma is often a process of trial and error, and is best done under the guidance of an enterostomal therapist. This is especially true for stomas in overweight individuals or stomas that have become flush with the skin with time and changing body habitus. Inattention to care can result in problems that range from simple mucocutaneous separations (separation of the bowel edge from the surrounding skin) to large and difficult-to-heal ulcers. This article provides a systematic review of the most common challenges that patients with stoma are faced with, and offers solutions based on up-to-date review of the literature.

Keywords: stoma care, stoma complications, stoma wound

\section{Introduction}

Enterostomy formation continues to have an important role in the treatment of colorectal and bladder cancer, constipation, pelvic floor dysfunction, and inflammatory bowel disease. ${ }^{1-9}$ In principle, a stoma is an artificial opening through which the bowel protrudes, and is attached to the skin surface, allowing stool or urine to be diverted to the outside of the body. ${ }^{4-6,9}$ The effluent must be separated from the skin, and contained within a pouching system that adheres to the skin. ${ }^{5}$ Keeping this system working well and healthy is critical in maintaining a good, well-functioning stoma. ${ }^{4,8,9}$

Keeping the peristomal skin intact proves to be a challenge for the patients, their caregivers, and the health care teams that work with them. Peristomal skin complications are shown to affect $18 \%-73 \%$ of patients. . $^{1,4,6,8-12}$ Ileostomies, and in particular loop ileostomies, are responsible for the greatest proportion of peristomal skin complications. ${ }^{1,2,4-6,11}$ Complications range from mild irritation to full thickness ulcerations leading to pain, anxiety, and in some cases significant social isolation related to the pouching system leaks. ${ }^{1,4,8,10}$ They are some of the most common postoperative 
complications, and usually occur within the first two weeks of the creation of the stoma. Peristomal skin complications can also present as late complications, months or even years after the initial surgery. ${ }^{2,7-11}$

Treatment should be tailored to the underlying cause of the complication; it is critical that the underlying cause is diagnosed, so an appropriate treatment regimen can be instituted. ${ }^{9}$ Treatments range from simply using different pouching systems, to a change in local care regimen, to administering systemic medications if the cause of the complications is related to a systemic disease process, and in some cases surgery may be necessary. ${ }^{1,2,4,8,9,13,14}$ This article provides an overview of the causes, effects, and treatments of peristomal skin complications.

\section{Causes of peristomal skin complications}

The causes of peristomal skin complications vary, though a common characteristic is leaking of the pouching system. ${ }^{1,8}$ In turn, there are a myriad of variables that can result in leaking. Structural characteristics such as stoma type, size, and location on the abdominal plane can affect the seal of the pouching system, and a poor fit can result in leakage and consequently irritation of the skin. ${ }^{1,2,6-8,11,15,16}$ Loop ileostomies represent the greatest proportion of peristomal skin complications in patients. In one study, $79 \%$ of the patients with a loop ileostomy suffered from a peristomal skin complication. ${ }^{11}$ Loop ileostomies often empty effluent close to the skin because the stoma itself frequently does not protrude sufficiently, ie, it is too flat. ${ }^{2}$ It is not uncommon for the loop ileostomy to further sink into the abdomen, more so than an end ileostomy would after the initial postsurgical edema recedes. ${ }^{1,2}$ Likely, having to make a larger defect in the fascia in order to allow two loops of bowel and the mesentery to be brought to the skin plays a role in this failure of the stoma to protrude sufficiently for a good seal. Having a low-lying stoma leads to effluent pooling near the stoma site, and eventual seeping underneath the flange; this is the opposite wanted result, where the stoma empties directly into the pouch away from the flange opening. ${ }^{1,2,11}$

End stomas are subject to similar technical challenges/ errors, and thus at risk to similar types of complications. Non-specialists may fashion a stoma that is insufficiently everted, and as the stoma shrinks (as the swelling recedes) after the first few weeks, it may recede at or below the skin level. ${ }^{1}$ A stoma that is on tension can put the blood supply and venous return at risk, in turn causing ischemia, and even if the stoma is ultimately viable, it often retracts. ${ }^{1,2,12}$
In obese patients, the mesentery of their stomas are thicker and shorter, making their stomas particularly at risk for these ischemic events that can result in a retracted stoma. ${ }^{1,2,6,15}$ The leakage is even more severe with a retracted stoma where the os is located beneath the abdominal plane. The pooling is so frequent that with an ileostomy in particular, the skin damage occurs quickly and often, and as the skin is exposed to the effluent rich in proteolytic enzymes, increasing the risk for these stomas to progress from simple irritation to advanced wounds. ${ }^{1,2,46,11}$

Even a well everted stoma can have significant leaking challenges if it is placed within a deep crease causing peristomal skin retraction circumferentially or only in one or two locations. Regardless of the extent of the retraction, any uneven areas of the peristomal plane can cause a place where effluent can pool and lead to a leak. . $^{1,2,7,15}$ These issues can occur with any patient; however, there is significantly greater risk of having an uneven peristomal plane in patients with a higher body mass index or if the patient was not evaluated by a stoma specialist preoperatively. 1,2,5-7,10,12,16 These dips and creases often occur immediately after surgery, but they can also occur years later, due to a parastomal hernia, weight gain, or weight loss. ${ }^{2,7,15}$ They can also occur due to mucocutaneous separations, localized infections, diabetes, and steroid usage. Mucocutaneous separations can be partial or circumferential, and frequently occur postoperatively; likely, they are due to issues with the technique of approximating the bowel to the skin. ${ }^{1,2}$

Patients may also suffer peristomal skin complications related to the pouching system products themselves. Mechanical trauma on peristomal skin or the hair follicles may occur due to repeated removal of the adhesive flanges and skin barrier products. Damage may even occur due to forceful and overly vigorous skin cleansing. ${ }^{24}$ Patients with sensitive skin or allergies may find that any number of the pouching system products may cause dermatitis. Flanges, paste, barrier rings, even the plastics of the drainable pouch clips may cause patients to have burning, redness, and at times weeping. Many times this allergic reaction leads to leaking and causes further denudement and ulceration., ${ }^{2,4}$

Additionally, peristomal skin complications may evolve from immunological or disease-related processes. Fungal infections are favored by the warm, moist climate that is found underneath the barrier flange, or can occur as an opportunistic infection if the patient is undergoing antibiotic therapy. Fungal infections have a unique pattern of satellite lesions formed by macules and papules. ${ }^{2,4}$ Peristomal fistulae present particularly difficult challenges, and there are multiple predisposing 
conditions that can lead to fistulae. Crohn's disease is a well-known risk for fistulae, and in a patient who has been diagnosed with ulcerative colitis and presents with fistulae, one must consider whether this is a case of Crohn's disease misdiagnosed as ulcerative colitis. ${ }^{1,4,7}$ Peristomal infection and abscess formation predisposes this area to fistulae formation. Peristomal pyoderma gangrenosum (PPG) is a painful and rare skin complication most often occurring in patients with inflammatory bowel disease..$^{2,4,7,13,14}$ PPG may occur any time after stoma formation, sometimes weeks to years later. Wounds found in PPG are extremely painful and typically present with pathergy where the wounds quickly escalate with the slightest trauma. ${ }^{2,4,7,13,14}$ All of these instances further cause difficulty with pouching adhesion and may become complicated by the effluent coming into contact with the skin.

\section{Effects of peristomal skin complications}

The majority of stoma research has focused on the causes of peristomal skin complications and how to treat them, but there are limited contemporary studies on the effects of peristomal skin complications. ${ }^{5,10}$ Most of the literature superficially discusses the three common effects: psychological and quality of life for the patient, medical community effects, and effects on society as a whole. In general, these studies focus on the full range of stoma complications, and their association with patient's decreased quality of life, as well as those involved in the patient's care. . $^{3,5,6,8,10,12,16-18}$

Sarkut et al specifically note that skin-related complications are the most common type of stoma complications, and these complications "considerably disrupt quality of life."12 Patients with peristomal skin complications must adapt to their new life, and frequently have long-lasting pain; in some cases patients become socially isolated. ${ }^{6,8,18}$ Changes in life can include reduced contact with family and friends, disruption in sleep, reduced general activity, and financial burden. ${ }^{6,10,18}$ Not only do these stressors cause anxiety and embarrassment, but also cause social isolation, which results in increased psychological strain. ${ }^{3}$

The patient is not the only individual affected by these issues. Peristomal skin complications and their sequelae cause challenges for society due to the financial toll, and impact on the health care system. ${ }^{6,10}$ In some cases patients will incur a significant financial burden for their supplies. Meisner et al determined that in severe cases of peristomal skin complications, costs for health care for a patient with a stoma increased from two to five times. ${ }^{10}$ Other studies conclude that peristomal skin complications increase these costs by $22 \% .{ }^{8}$ Patients may have to take extended leave from their place of employment, or may need to retire early. It is also found that peristomal skin complications account for $\sim 40 \%$ of all visits to stoma care nurses. ${ }^{8,10}$ Patients with skin difficulties report increased use of accessories, and may need help from stoma care nurses, which also increases the financial burden on patients and families. ${ }^{18}$ For patients with severe peristomal skin complications, systemic steroids or antibiotics are also factors that increase treatment costs significantly. ${ }^{10}$

\section{Treatment}

Treatment for the majority of these complications start with prevention. Proper stoma site selection and good surgical technique are arguably the most important factors of these preventative techniques. Preoperative stoma site marking performed by a trained enterostomy therapist or experienced surgeon greatly reduces the occurrence of peristomal skin complications. ${ }^{1,2,5-7,10,12,16}$ Any planned surgery in which stoma is likely, the patient should see a stoma specialist, not only for marking but also for preoperative counseling. Optimal site selection occurs within the abdominis rectus muscles as this reduces later complications, specifically parastomal hernias. The stoma site should be on a flat, smooth surface, at least $4 \mathrm{~cm}$ away from scars, skin folds, or bony prominences. The abdomen should be visualized in a number of body positions: the patient should be assessed while sitting, standing, lying down, and leaning forward. ${ }^{1,7}$ If the patient uses certain positions for work they should also be assessed mimicking these positions. The patient's belt line should be avoided if at all possible and ideally the stoma should be marked below the belt line. Patients with obese or pendulous abdomens may have to have their sites marked above the belt line because if the patient cannot properly visualize their stoma they will have difficulty pouching it. If the patient has a secondary stoma or will need a second stoma, the two sites should be marked off line from one another, in case a belt may be required for one or both of the pouches, as the stoma belts could cause trauma if overlapped on the opposing stoma. ${ }^{1,16}$

Excellent operative technique is also imperative. ${ }^{1,2,7,11}$ Taking care to create a stoma with a height over $10 \mathrm{~mm}$ ensures that the effluent empties directly into the pouch. This is important not only for the initial creation of the stoma but also from preventing vascular compromise. Any ischemia could lead to stomal retraction or mucocutaneous separation. To ensure the viability of the stoma, it is important to protect the segmental arterial supply to the stoma by reducing tension on the mesentery, and checking the tightness of the incision through 
the abdominal wall. Venous compromise may ensue from edema, or tension on the bowel mesentery. Obese patients offer challenges to ileostomy creation specifically. Bringing up a thickened mesentery through a thickened abdominal wall makes it difficult for the ileostomy to maintain adequate blood supply, and reach. Additionally, if there is tension on the mesentery, postoperatively the stoma will most likely be retracted. Assisting in the patient with weight loss if possible not only helps with the surgery, but also optimizes postoperative recovery. ${ }^{1,15}$ Finally, proper technique while suturing the stoma to the skin helps prevent mucocutaneous separations. ${ }^{2}$

Despite all preventative efforts, flush and retracted stomas and uneven peristomal planes do often occur. For flush or retracted stomas with flat, even peristomal planes, convexity is often enough to assist with maintaining an adequate seal between the skin and flange. If the patient has a softer abdomen firm convexity is advised, while a patient with a firm abdomen may do better with soft convexity. Flush stomas may need light convexity, while retracted stomas require a deeper convexity. For budded stomas with uneven peristomal pouching surfaces, caulking is necessary. Stoma paste, barrier paste strips, or barrier rings are used to fill in the dips and creases in order to create a flat pouching surface. When a patient presents with a short stoma as well as uneven peristomal planes, a mixture of these two techniques is needed. Convex rings or inserts may be combined with paste and strips in order to not only prevent areas of lifting but also prevent pooling and erosion of the opening of the flanges. ${ }^{2,8,11}$ For patients who develop peristomal hernias, the immediate peristomal plane may not be affected, but the protrusion of the abdomen even tangentially may cause difficulty with pouch adhesion. If the stoma is well budded and the immediate peristomal skin is flat and even, a hernia support belt may be enough to prevent separation. In the event of a hernia occurring with either a poor peristomal plane or small stoma, multiple techniques need to be employed in addition to the hernia belt. Likewise, stoma belts in conjunction with any of the aforementioned interventions may increase the efficacy in leak prevention for patients without hernias. Though regular $2.5 \mathrm{~cm}$ stoma belts should be adequate, in severe cases using a hernia support belt may be needed even if there is no hernia present. $^{2}$

Frequently, patients may already have skin damage at the time of the initial intervention. If damage is only slight redness, the use of a skin sealant may be enough to help heal the affected area. Areas of skin that already have partial thickness breakdown will need a skin barrier powder and then the use of skin sealant to provide an adhesive pouching surface.
This treatment is also effective for shallow mucocutaneous separations, although such separations may need multiple layers of the powder to skin sealant ratio. For peristomal ulcers that have depth or for mucocutaneous separations, often a hydrofiber or calcium alginate is needed to fill the dead space of the wound bed. ${ }^{2,9}$ In severe cases surgical intervention is required. ${ }^{1,2}$ If the patient has exhausted all materials and pouching system change routines, peristomal skin is severely damaged, and patient's quality of life is disabled, resiting of the stoma becomes necessary. Surgery should include preoperative site marking.

Mechanical and product-related damage are addressed in similar ways to leaking skin damage; education and product selection is essential, or the patient will continue to develop damage as the underlying cause of the damage is not being addressed. ${ }^{2,4}$ In situations where a patient suffers from a product-related skin reaction, applying layers of skin barrier powder and skin sealant protects the skin from coming into contact with the offending product. Although skin is healing from the irritant the patient should try and switch to a different brand, as different suppliers may use different materials. Skin damage due to flange removal can often be prevented by applying a layer of skin sealant so the plasticized layer is removed instead of skin. Proper technique by using adhesive removers and bracing the skin while pulling away the flange gently also minimizes damage caused by stripping. ${ }^{2,9}$ Patients may also clean the skin over-vigorously for fear of odor and infection. Although they believe that they are being vigilant by using soap or using copious amounts of friction, a flange may not adhere to residues left by the soap or to the damaged skin. If skin is damaged, skin barrier powders and skin sealant are effective in healing, but educating the patients in proper cleansing technique will also ensure that further damage does not occur. ${ }^{2}$ Although not related to the products themselves, patients may not measure flange openings correctly after surgery. As stomas naturally shrink in dimension due to the reduction of edema, patients may be cutting the flange opening to the original size of the stoma, which has retracted since surgery, and may leave the skin exposed to effluent. ${ }^{1,2,11}$ In instances of urostomies, the effect on the skin may not be ulceration but pseudoverrucous lesions. If encrustations are present due to the crystal formation, a vinegar soak is necessary, otherwise finding the right fit is the best method of healing the lesions. In severe cases the lesions might need to be shaved if pouching is truly difficult. ${ }^{4}$

Healing systemic disease and clearing up infections are the only ways to heal irritated skin from these derangements. 
Fungal infections clear rapidly with antifungal powders under the layer of skin sealants. If there is a mix of partial thickness ulcer with the maculopapular rash, barrier powder can be used in conjunction with an antifungal powder. ${ }^{2,8}$ For patients who present with fistulae and symptoms of Crohn's disease, discussions with the gastroenterology team is needed, as systemic therapy may be needed to help with the current fistula, and minimize the risk of another fistula formation. The fistula itself can be packed gently with calcium alginate or hydrofiber if large, but may do well with layering of barrier powder and skin sealant. For persistent fistulae the patient may have to undergo a resection of the peristomal disease and resiting of the stoma. ${ }^{1}$

Just as the above situations are treated with a mix of medications and wound care, so is PPG. Most importantly, care must be taken in the operating room to prevent the use of diseased bowel in making the stoma. ${ }^{2}$ Despite all precautions if the patient does develop PPG, meticulous wound care must go hand in hand with systemic treatments. There is no definitive diagnostic test for PPG, however, punch biopsies may reveal granulomas upon microscopic examination. ${ }^{2}$ Biopsies are to be done cautiously because the inflicted trauma may be enough to cause the degradation of the wound. Any trauma may induce the wound to progress deeper or larger. In addition to the quick deterioration of the wounds from the trauma, they may be painful. ${ }^{2,14}$ Corticosteroids are the medication of choice for treatment, and can be administered systemically or topically. When associated with Crohn's disease, tacrolimus - a $0.1 \%$ topical solution may be applied directly to the wound daily. Finally, in cases that are refractory to the above treatments, immunobiologics may need to be used. ${ }^{2,13,14}$ In terms of dressings for the lesions themselves, the optimal dressings must contain no adherent products to prevent the notorious pathergy. ${ }^{2,13,14}$

\section{Conclusion}

Peristomal skin complications have many implications for the patients and the care givers assisting them. Ranging from mild erythema to deep, full thickness ulceration, from contact dermatitis to PPG, peristomal skin complications have a wide range of causes, treatments, and effects on our society as a whole. ${ }^{10}$ Preventative measures are often just as important as the wound care treatments themselves, and the best way to ensure that complications are avoided is to partner with qualified and experienced health care providers for preoperative as well as postoperative care. Specially trained ostomy nurses may assist patients by not only treating complications of the stoma, but just as importantly, by helping the patients take good care of their stoma, and maintain a good fit of the products they use. ${ }^{2,11}$

With proper prevention and care, the quality of life of patients with stomas can be high. Anxiety, financial considerations, and disability can be assuaged with proper care and teamwork between medical staff, patient, and family. The field would benefit from more research in patient and caregivers-centered outcomes, and quality of life of the patients and their caregivers. Most importantly, implementation of well-established peristomal skin complications treatment techniques must be improved as peristomal skin complications rates have not drastically changed in the last 50 years. ${ }^{10}$

\section{Disclosure}

The authors report no conflicts of interest in this work.

\section{References}

1. Kann BR. Early stomal complications. Clin Colon Rectal Surg. 2008;21(1): 23-30.

2. Landmann RG. Routine care of patients with an ileostomy or colostomy and management of ostomy complications. Available from: http://www. uptodate.com/contents/routine-care-of-patients-with-an-ileostomyor-colostomy-and-management-of-ostomy-complications. Accessed August 18, 2016.

3. Liao C, Qin Y. Factors associated with stoma quality of life among stoma patients. Int Nurs Sci. 2014;1(2)196-201.

4. Lyon CC, Smith AJ, Griffiths CE, Beck MH. The spectrum of skin disorders in abdominal stoma patients. Br J Dermatol. 2000;143(6): 1248-1260.

5. Marquis P, Marrel A, Jambon B. Quality of life in patients with stomas: the Montreux Study. Ostomy Wound Manage. 2003;49(2):48-55

6. Pittman J, Rawl SM, Schmidt CM, et al. Demographic and clinical factors related to ostomy complications and quality of life in veterans with an ostomy. J Wound Ostomy Continence Nurs. 2008;35(5):493-503.

7. Shabbir J, Britton DC. Stoma complications: a literature overview. Colorectal Dis. 2010;12(1):958-964.

8. Szewczyk MT, Majewska G, Cabral MV, Hölzel-Piontek K. The effects of using a moldable skin barrier on peristomal skin condition in persons with an ostomy: results of a prospective, observational, multinational study. Ostomy Wound Manage. 2014;60(12):16-26.

9. Tam KW, Lai JH, Chen HC, et al. A systematic review and meta-analysis of randomized controlled trials comparing interventions for peristomal skin care. Ostomy Wound Manage. 2014;60(10):26-33.

10. Meisner S, Lehur PA, Moran B, Martins L, Jemec GB. Peristomal skin complications are common, expensive, and difficult to manage: a population based cost modeling study. PLoS One. 2012;7(5):e37813.

11. Persson E, Berndtsson I, Carlsson E, Hallén AM, Lindholm E. Stomarelated complications and stoma size - a 2-year follow up. Colorectal Dis. 2010;12(10):971-976.

12. Sarkut P, Dundar HZ, Tirnova I, Ozturk E, Yilmazlar T. Is stoma care effective in terms of morbidity in complicated ileostomies? Int J Gen Med. 2015;8:243-246.

13. Behm K, Larson DW, Colibaseanu D. Intravenous immunoglobulin use in managing severe, perioperative peristomal pyoderma gangrenosum following subtotal colectomy with end ileostomy for medically refractory chronic ulcerative colitis. J Surg Case Rep. 2015;2015(3):1-3.

14. Hughes AP, Jackson JM, Callen JP. Clinical features and treatment of peristomal pyoderma gangrenosum. JAMA. 2000;284(12): $1546-1548$. 
15. De Raet J, Delvaux G, Haentjens P, et al. Waist circumference is an independent risk factor for the development of parastomal hernia after permanent colostomy. Dis Colon Rectum. 2008;51(12):1806-1809.

16. Haugen V, Bliss DZ, Savik K. Perioperative factors that affect long-term adjustment to an incontinent ostomy. JWound Ostomy Continence Nurs. 2006;33(5):525-535.
17. Caricato M, Ausania F, Ripetti V, Bartolozzi F, Campoli G, Coppola R. Retrospective analysis of long-term defunctioning stoma complications after colorectal surgery. Colorectal Dis. 2007;9(6):559-561.

18. Erwin-Toth P. Prevention and management of peristomal skin complications. Adv Skin Wound Care. 2000;13(4):175-179.
Chronic Wound Care Management and Research

\section{Publish your work in this journal}

Chronic Wound Care Management and Research is an international, peer reviewed, open access, online journal publishing original research, reviews, editorials, and commentaries on the causes and management of chronic wounds and the major issues related to chronic wound management. Topics also include chronic wounds as comorbidities to other

Submit your manuscript here: https://www.dovepress.com/chronic-wound-care-management-and-research-journal
Dovepress

conditions, patient adherence to therapy, and the economic burden of chronic wounds. The manuscript management system is completely online and includes a very quick and fair peer review system, which is all easy to use. Visit http://www.dovepress.com/testimonials.php to read real quotes from published authors. 